Published in final edited form as:

Lancet Neurol. 2015 February ; 14(2): 224-234. doi:10.1016/S1474-4422(14)70160-7.

\title{
Constraint-Induced Movement Therapy after Stroke
}

\author{
Gert Kwakkel, $\mathrm{PhD}^{1,2}$, Janne M. Veerbeek, MSc ${ }^{1}$, Erwin E.H. van Wegen, $\mathrm{PhD}^{1}$, and Steven \\ L. Wolf, PhD, FPTA, FAHA ${ }^{3}$ \\ Janne M. Veerbeek: J.Veerbeek@vumc.nl; Erwin E.H. van Wegen: E.vanWegen@vumc.nl; Steven L. Wolf: \\ swolf@emory.edu
}

\begin{abstract}
${ }^{1}$ Department of Rehabilitation Medicine, MOVE Research Institute Amsterdam, VU University Medical Center; PO Box 7057; 1007 MB, Amsterdam, The Netherlands ${ }^{2}$ Amsterdam Rehabilitation Research Center, Reade Centre for Rehabilitation and Rheumatology, Amsterdam, The Netherlands ${ }^{3}$ Emory University School of Medicine, Department of Rehabilitation Medicine, Division of Physical Therapy, Atlanta VA Center for Visual and Neurocognitive Rehabilitation, 1441 Clifton Road NE; Atlanta, Georgia 30322 USA
\end{abstract}

\begin{abstract}
Constraint-induced movement therapy (CIMT) was developed to overcome upper limb impairments after stroke and is the most investigated intervention for treating stroke patients in the previous decades. This review describes the current evidence regarding: original CIMT and modified versions of CIMT (mCIMT). Meta-analysis showed strong evidence favoring both types of CIMT in terms of motor function, arm-hand activities and self-reported arm-hand functioning in daily life, immediately after treatment and at long-term follow-up, whereas no evidence was found for constraining alone (Forced Use (FU) therapy). No evidence was found that type of CIMT, intensity of practice or timing did affect outcome. Although the underlying mechanism that
\end{abstract}

\footnotetext{
(C) 2015 Elsevier Ltd. All rights reserved.

Corresponding author: G. Kwakkel, PhD; Department of Rehabilitation Medicine, MOVE Research Institute Amsterdam, VU University Medical Center; PO Box 7057; 1007 MB, Amsterdam, The Netherlands. Phone: +31-20-4440460; Fax: +31-20-4440787; G.Kwakkel@vumc.nl.

Authors' contributions to the manuscript: 1) Dr. G. Kwakkel: conceptualization of the study, interpretation of the data, drafting and revising the manuscript.

1) J.M. Veerbeek, MSc: conceptualization of the study, risk of bias assessment, performed the meta-analyses and interpretation of the data, revising the manuscript.

2) Dr. E.E.H. van Wegen: conceptualization of the study, risk of bias assessment, interpretation of the data, revising the manuscript.

3) Dr. S.L. Wolf: interpretation of the data, revising the manuscript.

Disclosure: Gert Kwakkel (PhD), Janne M. Veerbeek (MSc), Erwin E.H. van Wegen (PhD) and Steven L. Wolf (PhD) report no disclosures.

Conflicts of interest: GK has received grants from the European Research Council, Dutch National Institutes of Health (ZonMw), The Dutch Brain Foundation (Hersenstichting Nederland), The Dutch Hearth Foundation, and the Royal Dutch Society for Physical Therapy.

JV has received grants from the Royal Dutch Society for Physical Therapy. EvW has received grants from the Stichting Parkinson Fonds, the Beatrix Fonds, the Dutch Brain Foundation, Fonds Nuts-Ohra, the Dutch Parkinson Association, and ZonMw. SW has received grants from the National Institutes of Health (National Institute of Neurological Diseases and Stroke and the Center for Medical Rehabilitation Research within the National Institute of Child Health and Development).

Publisher's Disclaimer: This is a PDF file of an unedited manuscript that has been accepted for publication. As a service to our customers we are providing this early version of the manuscript. The manuscript will undergo copyediting, typesetting, and review of the resulting proof before it is published in its final citable form. Please note that during the production process errors may be discovered which could affect the content, and all legal disclaimers that apply to the journal pertain.
} 
drive (m)CIMT is still poorly understood, recent kinematic conducted studies suggests that improvements introduced by original CIMT or mCIMT are mainly based on adaptation by learning to optimize the use of intact end-effectors by selecting patients with some voluntary motor control of wrist and finger extensors post stroke.

\section{Keywords}

stroke; rehabilitation; upper limb; Constraint-induced movement therapy; forced use; systematic review; meta-analysis

\section{Introduction}

Worldwide, 16.9 million people suffer a first stroke each year, resulting in about 33 million stroke survivors and 5.9 million stroke-related death (1) making stroke the second or third most common cause of death and one of the main causes of acquired adult disability. $(1,2)$ Approximately $80 \%$ of these survivors have motor impairments of the upper limb(3) that gravely affect their ability to perform activities of daily living (ADL), as well as social participation. Previously we showed that the severity of upper limb paresis is an independent determinant of the outcome of basic activities of daily living (ADL) post stroke. (4)

A recent systematic review of 467 trials showed that the effectiveness of most interventions for the upper and lower limbs is driven by repetition and principles of task- and contextspecific motor learning. (5) Constraint-induced movement therapy (CIMT) or modified versions of CIMT (mCIMT) are currently considered the most effective treatment regimens in physical therapy to improve the outcome of the upper paretic limb. $(2,5)$

Although several systematic reviews concerning have been conducted in the past, (6-13) there is no recent meta-analysis of RCTs of (m)CIMT including thorough analyses of possible effect modifiers and small-study effects. Of the currently available reviews, some suffer from an incomplete literature search strategy, $(6,7,14)$ while others are restricted to a specific set of modified versions of CIMT(15), dose-matched controlled interventions(13), a specific period post stroke $(10,12)$, or a best-evidence synthesis based on the methodological quality of included trials. (11)

In the present review, first, we will briefly give a historical background and description of the original CIMT protocol. Based on a systematic review of the literature and subsequent metaanalysis of randomized controlled trials (RCTs), we will summarize the evidence for CIMT, mCIMT and Forced Use (FU) therapy in adult patients with stroke. In a subsequent sensitivity analysis of included RCTs, we will explore the effects of (1) CIMT type, (2) dosage of therapy, and (3) timing of therapy post stroke. Subsequently, we will discuss the effects of in light of assumed underlying mechanisms that drives (m)CIMT and criteria for selecting patients that will benefit most from (m)CIMT. Finally, we will summarize our findings and offer suggestions for further research.

The theoretical framework for CIMT has a long history.(16, 17) The German scientist Munk (1909) (18) was the first to document that non-human primates would use an impaired 
(deafferented) upper extremity if forced to do so, when the movement was purposeful and relevant. This work was quickly followed by the classical studies by Ogden and Franz (1917) who observed monkeys freely moving following pyramidal tract lesions.(19) Somewhat serendipitously, rather than by design, these animals were forced to use the hemiparetic upper extremity after immobilization of the better limb, which they were rapidly able to accomplish. This observation the authors to suggest that the limitation was not as much an inability as it was one of disuse. This concept of "forced use" (FU) was resurrected several decades later by Knapp(20) and in elegant studies by Taub(21), who applied the deafferented monkey model to show that these animals would not use the insensate limb unless a series of behavioral strategies was employed that resulted in 'overcoming learned non-use' (Fritz et al.(17) for more detail).

\section{Defining CIMT}

The signature protocol for the original form of CIMT contains three components or 'treatment packages': (1) intensive graded practice of the paretic upper limb aimed at enhancing task-specific use of the affected limb for up to 6 hours a day for 2 weeks (i.e., shaping) (Figure 1); (2) constraining or FU of the non-paretic upper limb with a mitt to promote the use of the more impaired limb during $90 \%$ of the waking hours; and (3) adherence-enhancing behavioral methods designed to transfer the gains obtained in the clinical setting or laboratory to the patients' real-world environment (i.e., transfer package). $(22,23)$ Thus, CIMT uses operant training techniques applied in the context of rehabilitation medicine, (24) while FU does not rely upon any conditioning. $(25,26)$ Since its introduction, the first proof of the original CIMT concept was investigated in 9 chronic stroke patients by Edward Taub and colleagues. (27) Their positive findings regarding motor function, dexterity and self-reported arm-hand use in daily life were later replicated in a multicenter trial of 222 stroke patients.(28-30) Trials by other research groups have applied "modified" forms of CIMT (mCIMT) that vary in dosage, timing and composition of therapy. While embracing the fundamental components of the original form of CIMT, these modifications are typically characterized by distributed training protocols with reduced time spent in training, less time during which the non-paretic upper limb is restrained, and absence of a transfer package including a contract with the patient, but a larger number of training days. $(31,32)$ Treatment sessions for mCIMT vary from 30 minutes(33-35) to 6 hours(36-44) a day, and from 2(45) to 7(46) sessions a week, for 2(23, 36-45, 47-56) to 12 weeks. The wide variety of these adaptations justifies a systematic review and subsequent meta-analysis of trials applying original or mCIMT. The definitions and description of rehabilitation terminology for (m)CIMT in the present review are summarized in supplementary web appendix 1.

\section{Effects of (m)CIMT}

CIMT has been investigated in 51 RCTs, $(23,28-31,33-82)$ including 1784 patients with adult stroke, but only 15 trials included patients within the first three months post stroke.(34, $45,47,49,50,52,53,56,59,66,67,69,76,78,82)$ Panel 1 shows the used search strategy and selection criteria used for this systematic review of the literature. For details about the search strategy, methods and flow-chart, see supplementary web appendix 2 to 4 . 


\section{Original CIMT}

Original CIMT, although seen as the 'gold standard' of CIMT, has been investigated in merely one RCT(28-30) and included patients beyond 3 months post stroke (see for details of the included trial of original CIMT supplementary web appendix 4). Significant positive medium effect sizes post intervention were found for arm-hand activities, self-reported amount of arm-hand use in daily life and self-reported quality of arm-hand movement in daily life as shown in Figure 2 (see also supplementary web appendix 6). The favorable effects of original CIMT for these three outcomes were sustained at follow-up (4 months) as shown in Figure 3 (see also supplementary web appendix 7). In addition, significant positive effect sizes in the long term were found for quality of life related to hand function and quality of life related to ADL.

\section{Modified versions of CIMT}

Contrary to original CIMT, mCIMT has been investigated in a vast amount of RCTs ( $\mathrm{n}=44$; $\mathrm{N}=1397$ ) (For details of included mCIMT trials see supplementary web appendix 4).(23, 31, 33-58, 61-64, 66, 70-78, 80-82) In favor of mCIMT, significant positive small to medium summary effect sizes (SESs) have been found post intervention in terms of motor function of the paretic arm, muscle tone, arm-hand activities, self-reported amount of arm-hand use in daily life, self-reported quality of arm-hand movement in daily life, and basic ADL (Figure 2) (see also supplementary web appendix 6). No significant summary effect sizes were found for grip strength, sensibility, pain, quality of life related to hand function, and quality of life related to ADL (Figure 2) (See supplementary web appendix 6). The effects were sustained at follow-up (mean 21.58 (SD 13.21) weeks) for motor function of the paretic arm, arm-hand activities, self-reported amount of arm-hand use in daily life, and selfreported quality of arm-hand movement in daily life, but not for muscle tone or basic ADL (Figure 3) (see also supplementary web appendix 7).

\section{Forced use}

Forced use therapy was investigated in 6 RCTs $(\mathrm{N}=165)(59,60,65,67-69,79)$ (see supplementary web appendix 5) and did not show an added value for self-reported amount of arm-hand use in daily life and self-reported quality of arm-hand movement in daily life (Figure 2) (see also supplementary web appendix 6).

\section{Impact of CIMT-type, dosage of therapy, and timing of therapy post stroke and small study effects}

Sensitivity analysis shows no significant differences in effect sizes between original and modified version of CIMT, dosage of (m)CIMT (additional time spent in exercise therapy $5(51)$ to 60 hours $(28,36,38,40,42)$ (mean 46.8 hours), and timing of (m)CIMT when comparing trials that started within or beyond the first 3 months post stroke. In addition, these robust effects for (m)CIMT seems not to be influenced by small-study effects, publication bias, nor were they moderated by risk of bias (supplementary web appendix 6 and 7). Despite not finding evidence for small-study effects, meta-regression of mCIMT trials showed that methodological quality was a significant effect modifier for motor function post intervention and self-reported use in daily life at follow-up. 


\section{What drives (m)CIMT?}

The underlying mechanism(s) that drive improvement by (m)CIMT are still poorly understood. Firstly, we expected that intensity of task-specific practice (expressed as treatment contrast in terms of duration) would be a significant moderator of (m)CIMT. However, as shown in this meta-analysis, we found no evidence that the type of (m)CIMT or treatment contrast - which amounted a mean of 47 hours - mattered. The absence of effects of treatment contrast between trials does not imply that dosing of CIMT therapy is not important. However, patients in (m)CIMT trials did practice much more intensively each day than is usual. In addition, Wolf and colleagues showed in a retrospective analysis of 169 participants that the intensity of supervised original CIMT was modified by the amount of repetitive task practice and also to some extent by the initial severity of motor impairment on the WMFT.(83) This finding suggests that the effects of the therapy dose are confounded by the initial severity of neurological deficits. Possible risks of bias, like blinding of assessors, did not seem to influence lack of difference between dose-matched trials and nondose matched trials. These findings are in agreement with a recent trial(82) and metaanalysis(84) showing that dose-matched trials of mCIMT in which the control group received an equal dose of bilateral arm training failed to report significant differences in overall effect sizes. Despite the finding that (m)CIMT may increase short-term $(50,85)$ and long-term cortical activation patterns, $(42,50,86)$, the underlying mechanisms responsible for improvements require further investigation. In particular, uncertainty continues to exist about how observed improvements in motor performance after (m)CIMT relate to recruitment of cortical activation patterns in the contralesional and ipsilesional cortex after stroke, as revealed by Transcranial Magnetic Stimulation (TMS) $(42,86,87)$ and functional Magnetic Resonance Imaging (fMRI) (88-90) (See for a critical review: Buma et al. (91)). For example, recent studies suggest that improved hand function assessed by WMFT is accompanied by increased recruitment of ipsilesional somatosensory cortex. $(89,90)$ Although significant neural correlates have been found with upper extremity measurements, such as WMFT, these studies do not address the question how cortical changes relate to the quality of motor performance in terms of 'neural repair' or using compensation strategies. (See for a review: Buma et al.(92)) For example, a recent controlled proof of concept study of Kitago and colleagues failed to show significant changes in coordinative measures of the paretic arm and wrist after (m)CIMT in chronic stroke, despite clinically meaningful functional improvements in ARAT scores.(93) (See also references (33, 35, 37, 38, 43, 46, $57,62,64,70-72,80)(35))$ This observation further suggests that improvements introduced by original CIMT or mCIMT are mainly based on learning to optimize the use of intact endeffectors (i.e., compensation strategies). These findings also suggest that the enhanced cortical neuroplasticity found by $\operatorname{TMS}(42,86,87)$ and fMRI $(90)$ in the subacute $(49,50)$ and chronic post stroke phases $(42,87)$ may reflect learned non-use and compensatory skill learning rather than "true neurological repair" or "recovery" of impairments (see also references $(14,92,94))$. This assumption is further supported by some recent longitudinal 3D-kinematic studies showing that the number of degrees of freedom that patients can engage while performing meaningful tasks, such as reaching, is mainly completed in the first 8 weeks post stroke. $(94,95)$ These improvements in intra-limb coordination are accompanied by a significant reduction in patients' variability(94) and improvement in the 
smoothness(95) of their motor performance. Interestingly, the 3D-kinematic improvements closely follow the clinical time course of impairments such as synergism $(94,96,97)$ and are also restricted to the first 3 months post stroke. $(98,99)$ Interestingly, the present metaanalysis further suggests that the effects of mCIMT on motor function of the arm such as FMA-arm scores is restricted to trials that started within this time window of 3 months post stroke (Table 1 and supplementary web appendix 6 and 7) (References $(34,45,49,50,82)$ ). This finding is in agreement with the growing evidence from animal studies in which the first weeks after stroke onset are characterized by increased levels of homeostatic neuroplasticity (100)

\section{Who should be selected for $(\mathrm{m}) \mathrm{CIMT}$ ?}

An important inclusion criterion for the original CIMT trial was that patients showed some voluntary extension at the wrist and some minimal extension at the metacarpophalangeal and interphalangeal joints at baseline.(28) Within this selection criterion one may discriminate between higher-functioning participants who demonstrate at least $20^{\circ}$ of wrist extension and at least $10^{\circ}$ of active extension of each metacarpophalangeal and interphalangeal joint of all digits, and lower-functioning participants who shows at least $10^{\circ}$ of active wrist extension, at least $10^{\circ}$ of thumb abduction/extension, and at least $10^{\circ}$ of extension in at least 2 additional digits. Preferably, these movements had to be repeated 3 times in 1 minute. (101)

Although severity of stroke was not tested formally in this review, the ability to extend one or more fingers of the paretic side seems to be natural due to the fact that active repetition of movements and activities is not possible when there is no function at all. Recent transcranial magnetic stimulation (TMS) (86) and diffusion tensor imaging (DTI) $(102,103)$ studies have shown that voluntary wrist and, particularly, finger extension are highly associated with the integrity of the corticospinal tract (CST) system. This motor function is the strongest clinical predictor of the return of some dexterity in the first days post stroke. (103-106) Accordingly, Fritz and colleagues showed in 55 chronic stroke subjects that initial presence of finger extension was the only significant predictor of outcome of the WMFT after applying original CIMT. (107) Accordingly, the selection of patients with some extension of wrist and fingers should be seen as a key factor determining the potential for change $(103,105)$ and reversal of learned non-use by CIMT after stroke. (107) In addition, due to concerns about the safety of the restraint by a sling or splint applied in the original form of CIMT, (36) which might prevent adequate protective reactions to control standing balance, the restraint has been replaced by a padded mitt,(108) and patients should be able to stand for at least 2 minutes with or without support. (16) Other, more general criteria, included a Mini-Mental State Examination (MMSE) score of $\geq 24$, no major medical problems that could interfere with participation, no history of previous stroke, no excessive pain or spasticity in the paretic extremity, enough stamina to participate, and age above 18 years. (16) Collectively, these inclusion criteria suggest that (m)CIMT is best restricted to patients with a mild to moderate paresis with a predominantly favorable prognosis for dexterity early post stroke. To date, about $10 \%$ (range 3(53) to 90\%(59)) of the initially screened patients of included trials in the present review was eligible for (m) CIMT. 


\section{Synthesizing current evidence of $(\mathrm{m}) \mathrm{CIMT}$}

From systematic review of the literature and subsequent meta-analyses it is clear that modified versions of CIMT ( $\mathrm{n}=44)$ and FU $(\mathrm{n}=6)$ have been investigated in several mainly small, underpowered trials, whereas original CIMT has only been investigated in one sufficiently powered landmark trial. (28) Despite the heterogeneity among the forms of mCIMT applied, meta-analyses show that original and modified versions of CIMT have a robust, clinically meaningful impact on patient's outcomes for arm-hand activities, selfreported amount and quality of arm-hand use in daily life, and basic ADL, making (m)CIMT one of the most effective interventions for the upper paretic limb post stroke. (5) For example, an anchor-based change of 12 to 17 points (21-30\%) in dexterity following the ARAT is considered as 'clinically important' or 'meaningful' in patients measured within the first month post stroke. (109), whereas in chronic stroke victims, a distribution-based change of about 6 points (10\%) is found as clinically meaningful on the ARAT (110). This finding further emphasizes that the minimal clinically important difference (MCID) of used upper extremity measures such as ARAT and WMFT are not only context specific but also dynamic in time.(109)

With the exception of muscle tone and basic ADL, the significant positive effects were sustained in the longer term, even though the size of SESs decreased after ending (m)CIMT. In addition, original CIMT showed evidence of benefits in long-term health-related quality of life.(28)

This review also suggests that (m)CIMT has no significant effects on grip strength, sensibility, pain, or health-related quality of life post intervention. However, the statistical power underpinning the evidence was limited by the insufficient numbers of patients in (m)CIMT trials using these outcomes. A summary of findings in terms of body functions, activities and participation is shown in Table 1 .

This review also suggests that, based on RCTs in which the only difference between the experimental and control groups was wearing a mitt on the less affected arm without a structured exercise program (i.e. FU), no superiority to the outcome was found. This finding suggests that procedures involving shaping, repetitive exercises and instructions for behavioural change are the most important components of (m)CIMT. Despite the large number of trials identified, sensitivity analyses showed no significant differences between types of CIMT regimen, timing of (m)CIMT post stroke, or treatment contrast between experimental and control groups.

Overall, the methodological quality or treatment contrast did not significantly affect our results, however small mCIMT trials with lower methodological quality did significantly overestimate the post intervention scores on motor function, while self-reported amount of arm-hand use in daily life showed overestimation of its effect sizes in the long term (see supplementary web appendix 6).

These findings further extend the knowledge of the effectiveness of CIMT and hypothetical accompanying mechanisms from previous reviews, $(6-10,12,13,15,111)$ by determining the effects and especially their sustainability on all domains of the ICF, based on well- 
powered meta-analyses. The post intervention effects on the activities level were confirmed, but we the present review also shows that the effects are maintained for at least 4-5 months after termination of the intervention. In addition, the present review showed strong evidence that CIMT has greater effects on motor function only when applied in the earlier stages post stroke, in which it is assumed that restitution of neurological functions is still possible, but that in the later phases CIMT solely influences arm-hand activities by learning to use adaptation strategies (i.e., compensation) to improve upper limb performance in ADLs.(14)

\section{Limitations}

The present systematic review has some limitations. First, we could only explore differential effects between original type of CIMT and mCIMT by using forest plots. However, the 44 mCIMT trials are heterogeneous in terms of content and intensity. Second, although we did not detect common threats to meta-analyses from small-study effects or publication bias, (112) we may have missed small negative trials. We synthesized only aggregate study level data obtained from cited studies of sufficient methodological quality (i.e., PEDro > 4). Including the 5 trials with low methodological quality would not have affected significantly the overall medium sized effects and conclusions in the present review. Unfortunately, we were unable to perform meta-analysis of individual patient data.(113) As a consequence, we were unable to investigate possible effect modifiers, such as arm dominance and the influence of cognitive limitations such as dyspraxia, age, or type of stroke. To investigate long-term effects, we pooled data from trials with different follow-up intervals. Also, our meta-analyses of measures such as grip strength and health-related quality of life were underpowered, so the effect of (m)CIMT on these outcomes remains unclear. Our sensitivity analyses should be interpreted with caution; due to uneven distribution across subgroups and in some cases inclusion of only one trial in a subgroup, these analyses should mainly be seen as indications.(114)

Analyses of the statistical power of pooled trials showed that about half of the statistical analyses for (m)CIMT and FU post intervention and in the longer term are sufficiently powered (supplementary web appendix 5 and 6 ). The lack of statistical power applies more to the pooled trials that started within the first three months post stroke, as well for those studies that investigated the sustainability of (m)CIMT.

Finally, the optimal dosage of (m)CIMT remains unclear, but should range between the numbers as described in the section 'The CIMT protocol'. Although not tested formally in this review due to the lack of a sufficient number of RCTs, the use of a transfer package to enhance intensity of practice could be considered.

\section{Future directions}

The present review shows that only 15 out of the 51 trials provided mCIMT within the first weeks post stroke, whereas all these RCTs were small, phase II trials. This finding suggests that more mCIMT trials are needed preferably starting within the first days post stroke using different doses of upper limb training. There is growing evidence from animal studies that the brain shows increased neuroplasticity in the early phases post stroke. This finding suggests that normalization of motor control by true neurological recovery could be 
maximized within this time window. $(92,93,100)$ Results from several animal studies(100, 115-117) suggest that (m) CIMT in the first weeks after stroke may enhance up-regulation of growth promoting factors such as protein 43 , synaptophysin and other brain derived neurotrophic factors. (117) In addition, Zhao and colleagues recently showed that applying (m) CIMT from weeks 1 to 3 post stroke significantly suppressed the up-regulation of growth inhibiting factors such as Nogo-A, Nogo receptors and RhoA expressed in the periinfarct cortex in Wistar rats.(117) In these animals, mCIMT resulted in significant structural post-synaptic plastic changes in the denervated cervical spinal cord. (117) Applying mCIMT for 4 weeks directly after stroke fosters reorganization of the somatosensory cortex and its neural network.(118) One emerging question is whether the structural plasticity introduced by early applied mCIMT also leads to true neurological repair beyond the existing mechanisms of spontaneous neurological recovery in the first phase post stroke.(92) The limited time window of neural mechanisms assumed to play a role in the natural logarithmic pattern of spontaneous neurological recovery of body functions (or reduction in impairments) may further underline the need for more RCTs with intensive serial assessments in time early post stroke. Preferably, to improve our understanding of skill acquisition by mCIMT, these observed improvements in repeated assessments should be associated with serial measures of kinematics, biomechanics and non-invasive neuroimaging techniques post stroke. $(2,92)$

Second, there is a need to investigate the assumptions of learned "misuse" when patients learn to use their end-effectors in a different adaptive way to normalize motor control early post stroke.(119) Such research should objectively and intensively monitor the quality of motor control in terms of temporal-spatial activation patterns of the upper limb and trunk using 3D-kinematics and EMG-controlled measures in addition to complementary clinical outcomes. (92) This approach would allow investigation of changes in end-effectors of the paretic arm and trunk during stroke recovery. (94) Preferably, these coordination measures should be related to neuronal correlates to allow appropriate interpretation of changes in neuroplasticity observed in animal studies. $(92,120,121)$

Third, additional research is needed to investigate possible detrimental effects of very high doses of early applied (m)CIMT (i.e., > 3 hours) within this time window of increased homeostatic neuroplasticity, as suggested by some studies among animals (122-124) and patients with stroke. (53) Interestingly, a recent meta-analysis involving 8 animal trials showed no significant inverse dose-response relationship of (m)CIMT on infarct volume $(-3 \%, 95 \%$ CI 15-9; $\mathrm{p}=\mathrm{p}=0.63) .(121)$ This finding further emphasizes that animal models may help us to efficiently explore the biological basis of rehabilitation interventions, but also questions its generalizability to humans. (121)

Fourth, none of the identified trials reported the influence of phenotypic factors such as gender, age or type of stroke on the effects of (m) CIMT on outcome after stroke. One trial claimed larger effects for chronic stroke patients with sensory deficits and neglect. (37) Investigating the relation between individual patient characteristics and the effects of (m)CIMT requires meta-analysis of individual patient data to identify possible effect modification by patients' phenotypes.(113) 
Fifth, most mCIMT trials lack transparency in the treatment protocol with respect to content, timing post stroke and therapy dosage. Fortunately, most journals now accept online submission of treatment protocols applied in trials. In addition, consensus is needed on the content and timing of test batteries applied to evaluate (m) CIMT. (2)

Finally, the barriers to implementation of (m)CIMT and factors which may enhance realworld use of the upper paretic limb require further investigation. (23) In view of the limited health care resources in most countries, and the increasing numbers of stroke survivors, there is a need to investigate the cost-effectiveness of (m)CIMT compared to usual care. (16) Adding therapy time will result in a concomitant increase in health-care costs, however effective therapy could potentially reduce rates of readmission to hospitals and admission to long-term care institutions.(125) In addition, innovative, adaptive forms of (m)CIMT, such as group sessions to reduce the staff-to-patient ratio and costs, self-training mCIMT programs (e.g., (126)), caregiver-support, and supervised practice by e-health support and telerehabilitation services, need to be investigated and compared with the usual face-to-face (m)CIMT regarding cost-benefit.(11)

\section{Supplementary Material}

Refer to Web version on PubMed Central for supplementary material.

\section{Acknowledgments}

The authors would like to thank Hans Ket (Librarian VUmc) for his cooperation in the literature search and Mark van den Brink for the illustration. The present study was funded by a grant from the Royal Dutch Society of Physiotherapy (grant no. 8091.1) and supported by the EXPLICIT-stroke grant from The Netherlands Organisation for Health Research and Development (ZonMw) (grant no. 89000001) and 4D-EEG (ERC advanced grant no. 291339-4D-EEG). We thank Paul Thompson PhD for providing additional EXCITE data used in the original CIMT analyses.

The funding organizations had no role in the design or conduct of the study, the collection, management, analysis, or interpretation of the data, or the preparation, review, or approval of the manuscript.

Study funding: The present study was funded by a grant from the Royal Dutch Society of Physiotherapy (/grant no. 8091.1; http://www.fysionet.nl), supported by the EXPLICIT-stroke grant from the Netherlands Organisation for Health and development (ZonMw; grant no. 89000001; http://www.zonmw.nl; http://www.explicit-stroke.nl), and 4D-EEG (ERC advanced grant no. 291339-4D-EEG; http://erc.europa.eu/). The funding organizations had no role in the design or conduct of the study or the collection, management, analysis, or interpretation of the data; or the preparation, review, or approval of the manuscript.

\section{References}

1. Feigin V, Faorouzanfar M, Krishamurthi R, Mensah G, Connor M, Bennett D, et al. Global and regional burden of stroke during 1990 - 2010: findings from the Global Burden of Disease Study 2010. Lancet. 2013

2. Langhorne P, Bernhardt J, Kwakkel G. Stroke rehabilitation. Lancet. 2011; 377(9778):1693-702. [PubMed: 21571152]

3. Langhorne P, Coupar F, Pollock A. Motor recovery after stroke: a systematic review. Lancet Neurol. 2009; 8(8):741-54. [PubMed: 19608100]

4. Veerbeek J, Kwakkel G, van Wegen E, Ket J, Heymans M. Early prediction of outcome of activities of daily living after stroke: a systematic review. Stroke. 2011; 42(5):1482-8. [PubMed: 21474812]

5. Veerbeek J, van Wegen E, van Peppen R, van der Wees P, Hendriks H, Rietberg M, et al. What is the evidence for physical therapy poststroke? A systematic review and metaanalysis. Submitted. 
6. Sirtori V, Corbetta D, Moja L, Gatti R. Constraint-induced movement therapy for upper extremities in stroke patients. Cochrane Database Syst Rev. 2009; (4):CD004433. [PubMed: 19821326]

7. Corbetta D, Sirtori V, Moja L, Gatti R. Constraint-induced movement therapy in stroke patients: systematic review and meta-analysis. European journal of physical and rehabilitation medicine. 2010; 46(4):537-44. [PubMed: 21224785]

8. Hakkennes S, Keating J. Constraint-induced movement therapy following stroke: a systematic review of randomised controlled trials. The Australian journal of physiotherapy. 2005; 51(4):22131. [PubMed: 16321129]

9. Bonaiuti D, Rebasti L, Sioli P. The constraint induced movement therapy: a systematic review of randomised controlled trials on the adult stroke patients. Europa medicophysica. 2007; 43(2):13946. [PubMed: 17525700]

10. Nijland R, Kwakkel G, Bakers J, van Wegen E. Constraint-induced movement therapy for the upper paretic limb in acute or sub-acute stroke: a systematic review. International journal of stroke: official journal of the International Stroke Society. 2011; 6(5):425-33. [PubMed: 21951408]

11. Reiss A, Wolf S, Hammel E, McLeod E, Williams E. Constraint-Induced Movement Therapy (CIMT): Current Perspectives and Future Directions. Stroke research and treatment. 2012; 2012:159391. [PubMed: 22577601]

12. McIntyre A, Viana R, Janzen S, Mehta S, Pereira S, Teasell R. Systematic review and metaanalysis of constraint-induced movement therapy in the hemiparetic upper extremity more than six months post stroke. Top Stroke Rehabil. 2012; 19(6):499-513. [PubMed: 23192715]

13. Stevenson T, L T, Christie H, Poluha W. Constraint-Induced Movement Therapy Compared to Dose-Matched Interventions for Upper-Limb Dysfunction in Adult Survivors of Stroke: A Systematic Review with Meta-analysis. Physiotherapy Canada Physiotherapie Canada. 2012; 64(4):397-413. [PubMed: 23997396]

14. Sunderland A, Tuke A. Neuroplasticity, learning and recovery after stroke: a critical evaluation of constraint-induced therapy. Neuropsychological rehabilitation. 2005; 15(2):81-96. [PubMed: 16353503]

15. Shi Y, Tian J, Yang K, Zhao Y. Modified constraint-induced movement therapy versus traditional rehabilitation in patients with upper-extremity dysfunction after stroke: a systematic review and meta-analysis. Arch Phys Med Rehabil. 2011; 92(6):972-82. [PubMed: 21621674]

16. Viana R, Teasell R. Barriers to the implementation of constraint-induced movement therapy into practice. Top Stroke Rehabil. 2012; 19(2):104-14. [PubMed: 22436358]

17. Fritz S, Butts R, Wolf S. Constraint-induced movement therapy: from history to plasticity. Exp Rev Neurother. 2012; 12(2):191-98.

18. Munk, H. Ueber die functionen von hirn und ruckenmark. Berlin: Hirshwald; 1909.

19. Ogden R, Franz S. On cerebral motor control: the recovery from experimentally produced hemiplegia. Psychobiol. 1917; 1(1):33-50.

20. Knapp H, Taub E, Berman A. Movements in monkeys with deafferented forelimbs. Experimental neurology. 1963; 7:305-15. [PubMed: 14033714]

21. Taub, E. Somatosensory deafferentation research with monkeys: implications for rehabilitation medicine. In: Ince, L., editor. Behavioral Psychology in Rehabilitation Medicine: Clinical Applications. Baltimore: Williams and Wilkins; 1980. p. 371-401.

22. Morris D, Taub E, Mark V. Constraint-induced movement therapy: characterizing the intervention protocol. Europa medicophysica. 2006; 42(3):257-68. [PubMed: 17039224]

23. Taub E, Uswatte G, Mark V, Morris D, Barman J, Bowman M, et al. Method for enhancing realworld use of a more affected arm in chronic stroke: transfer package of constraint-induced movement therapy. Stroke. 2013; 44(5):1383-8. [PubMed: 23520237]

24. Taub E. The behavior-analytic origins of constraint-induced movement therapy: an example of behavioral neurorehabilitation. The Behavior analyst / MABA. 2012; 35(2):155-78. [PubMed: 23449867]

25. Ostendorf C, Wolf S. Effect of forced use of the upper extremty of a hemiplegic patient on changes in function. A single case design. Phys Ther. 1981; 61(7):1022-28. [PubMed: 7243897] 
26. Wolf S, Lecraw D, Barton L, Jann B. Forced use of hemiplegic upper extremities to reverse the effect of learned nonuse among chronic stroke and head-injured patients. Experimental neurology. 1989; 104(2):125-32. [PubMed: 2707361]

27. Taub E, Crago J, Burgio L, Groomes T, Cook E 3rd, DeLuca S, et al. An operant approach to rehabilitation medicine: overcoming learned nonuse by shaping. Journal of the experimental analysis of behavior. 1994; 61(2):281-93. [PubMed: 8169577]

28. Wolf S, Winstein C, Miller J, Taub E, Uswatte G, Morris D, et al. Effect of constraint-induced movement therapy on upper extremity function 3 to 9 months after stroke: the EXCITE randomized clinical trial. JAMA. 2006; 296(17):2095-104. [PubMed: 17077374]

29. Wolf S, Winstein C, Miller J, Thompson P, Taub E, Uswatte G, et al. Retention of upper limb function in stroke survivors who have received constraint-induced movement therapy: the EXCITE randomised trial. Lancet Neurol. 2008; 7(1):33-40. [PubMed: 18077218]

30. Wolf S, Thompson P, Winstein C, Miller J, Blanton S, Nichols-Larsen D, et al. The EXCITE stroke trial: comparing early and delayed constraint-induced movement therapy. Stroke. 2010; 41(10):2309-15. [PubMed: 20814005]

31. Page S, Sisto S, Levine P, Johnston M, Hughes M. Modified constraint induced therapy: a randomized feasibility and efficacy study. J Rehabil Res Dev. 2001; 38(5):583-90. [PubMed: 11732835]

32. Woodbury, M.; Fritz, S.; Blanton, S.; Wolf, S. Chapter 1: History of the development of CIMT. In: Ramey, S.; Coker-Bolt, P.; Deluca, S., editors. The Handbook of Pediatric Constraint-Induced Movement Therapy (P-CIMT): Translating Theory into Clinical Practice and Functional Occupations. Bethesda: American Occupational Therapy Association; 2013. p. 3-19.

33. Page S, Sisto S, Levine P, McGrath R. Efficacy of modified constraint-induced movement therapy in chronic stroke: a single-blinded randomized controlled trial. Arch Phys Med Rehabil. 2004; 85(1):14-8. [PubMed: 14970962]

34. Page S, Levine P, Leonard A. Modified constraint-induced therapy in acute stroke: a randomized controlled pilot study. Neurorehabil Neural Repair. 2005; 19(1):27-32. [PubMed: 15673841]

35. Page S, Levine P, Leonard A, Szaflarski J, Kissela B. Modified constraint-induced therapy in chronic stroke: results of a single-blinded randomized controlled trial. Phys Ther. 2008; 88(3): 333-40. [PubMed: 18174447]

36. Taub E, Miller N, Novack T, Cook E 3rd, Fleming W, Nepomuceno C, et al. Technique to improve chronic motor deficit after stroke. Arch Phys Med Rehabil. 1993; 74(4):347-54. [PubMed: 8466415]

37. van der Lee J, Wagenaar R, Lankhorst G, Vogelaar T, Deville W, Bouter L. Forced use of the upper extremity in chronic stroke patients: results from a single-blind randomized clinical trial. Stroke. 1999; 30(11):2369-75. [PubMed: 10548673]

38. Alberts J, Butler A, Wolf S. The effects of constraint-induced therapy on precision grip: a preliminary study. Neurorehabil Neural Repair. 2004; 18(4):250-8. [PubMed: 15537995]

39. Suputtitada A, Suwanwela N, Tumvitee S. Effectiveness of constraint-induced movement therapy in chronic stroke patients. J Med Assoc Thai. 2004; 87(12):1482-90. [PubMed: 15822545]

40. Yen J, Wang R, Chen H, Hong C. Effectiveness of modified constraint-induced movement therapy on upper limb function in stroke subjects. Acta Neurol Taiwan. 2005; 14(1):16-20. [PubMed: 15835284]

41. Dahl A, Askim T, Stock R, Langorgen E, Lydersen S, Indredavik B. Short- and long-term outcome of constraint-induced movement therapy after stroke: a randomized controlled feasibility trial. Clin Rehabil. 2008; 22(5):436-47. [PubMed: 18441040]

42. Sawaki L, Butler A, Leng X, Wassenaar P, Mohammad Y, Blanton S, et al. Constraint-induced movement therapy results in increased motor map area in subjects 3 to 9 months after stroke. Neurorehabil Neural Repair. 2008; 22(5):505-13. [PubMed: 18780885]

43. Woodbury M, Howland D, McGuirk T, Davis S, Senesac C, Kautz S, et al. Effects of trunk restraint combined with intensive task practice on poststroke upper extremity reach and function: a pilot study. Neurorehabil Neural Repair. 2009; 23(1):78-91. [PubMed: 18812433] 
44. Hayner K, Gibson G, Giles G. Comparison of constraint-induced movement therapy and bilateral treatment of equal intensity in people with chronic upper-extremity dysfunction after cerebrovascular accident. Am J Occup Ther. 2010; 64(4):528-39. [PubMed: 20825123]

45. Singh P, Pradhan B. Study to assess the effectiveness of modified constraint-induced movement therapy in stroke subjects: A randomized controlled trial. Annals of Indian Academy of Neurology. 2013; 16(2):180-4. [PubMed: 23956560]

46. Abu Tariah H, Almalty A, Sbeih, Al-Oraibi S. Constraint induced movement therapy for stroke survivors in Jordon: a home-based model. Int J Ther Rehab. 2010; 17(12):638-46.

47. Dromerick A, Edwards D, Hahn M. Does the application of constraint-induced movement therapy during acute rehabilitation reduce arm impairment after ischemic stroke? Stroke. 2000; 31(12): 2984-8. [PubMed: 11108760]

48. Wittenberg G, Chen R, Ishii K, Bushara K, Eckloff S, Croarkin E, et al. Constraint-induced therapy in stroke: magnetic-stimulation motor maps and cerebral activation. Neurorehabil Neural Repair. 2003; 17(1):48-57. [PubMed: 12645445]

49. Ro T, Noser E, Boake C, Johnson R, Gaber M, Speroni A, et al. Functional reorganization and recovery after constraint-induced movement therapy in subacute stroke: case reports. Neurocase. 2006; 12(1):50-60. [PubMed: 16517515]

50. Boake C, Noser E, Ro T, Baraniuk S, Gaber M, Johnson R, et al. Constraint-induced movement therapy during early stroke rehabilitation. Neurorehabil Neural Repair. 2007; 21(1):14-24. [PubMed: 17172550]

51. Gauthier L, Taub E, Perkins C, Ortmann M, Mark V, Uswatte G. Remodeling the brain: plastic structural brain changes produced by different motor therapies after stroke. Stroke. 2008; 39(5): 1520-5. [PubMed: 18323492]

52. Myint J, Yuen G, Yu T, Kng C, Wong A, Chow K, et al. A study of constraint-induced movement therapy in subacute stroke patients in Hong Kong. Clin Rehabil. 2008; 22(2):112-24. [PubMed: 18212033]

53. Dromerick A, Lang C, Birkenmeier R, Wagner J, Miller J, Videen T, et al. Very Early ConstraintInduced Movement during Stroke Rehabilitation (VECTORS): A single-center RCT. Neurology. 2009; 73(3):195-201. [PubMed: 19458319]

54. Huseyinsinoglu B, Ozdincler A, Krespi Y. Bobath Concept versus constraint-induced movement therapy to improve arm functional recovery in stroke patients: a randomized controlled trial. Clin Rehabil. 2012; 26(8):705-15. [PubMed: 22257503]

55. Smania N, Gandolfi M, Paolucci S, Iosa M, Ianes P, Recchia S, et al. Reduced-intensity modified constraint-induced movement therapy versus conventional therapy for upper extremity rehabilitation after stroke: a multicenter trial. Neurorehabil Neural Repair. 2012; 26(9):1035-45. [PubMed: 22661278]

56. Treger I, Aidinof L, Lehrer H, Kalichman L. Modified constraint-induced movement therapy improved upper limb function in subacute poststroke patients: a small-scale clinical trial. Top Stroke Rehabil. 2012; 19(4):287-93. [PubMed: 22750958]

57. Page S, Sisto S, Johnston M, Levine P. Modified constraint-induced therapy after subacute stroke: a preliminary study. Neurorehabil Neural Repair. 2002; 16(3):290-5. [PubMed: 12234091]

58. Atteya A. Effects of modified constraint induced therapy on upper limb function in subacute stroke patients. Neurosciences (Riyadh). 2004; 9(1):24-9. [PubMed: 23377299]

59. Ploughman M, Corbett D. Can forced-use therapy be clinically applied after stroke? An exploratory randomized controlled trial. Arch Phys Med Rehabil. 2004; 85(9):1417-23. [PubMed: 15375810]

60. Brogärdh C, Sjolund B. Constraint-induced movement therapy in patients with stroke: a pilot study on effects of small group training and of extended mitt use. Clin Rehabil. 2006; 20(3):218-27. [PubMed: 16634340]

61. Lin K, Wu C, Wei T, Lee C, Liu J. Effects of modified constraint-induced movement therapy on reach-to-grasp movements and functional performance after chronic stroke: a randomized controlled study. Clin Rehabil. 2007; 21(12):1075-86. [PubMed: 18042603] 
62. Wu C, Chen C, Tang S, Lin K, Huang Y. Kinematic and clinical analyses of upper-extremity movements after constraint-induced movement therapy in patients with stroke: a randomized controlled trial. Arch Phys Med Rehabil. 2007; 88(8):964-70. [PubMed: 17678656]

63. Wu C, Chen C, Tsai W, Lin K, Chou S. A randomized controlled trial of modified constraintinduced movement therapy for elderly stroke survivors: changes in motor impairment, daily functioning, and quality of life. Arch Phys Med Rehabil. 2007; 88(3):273-8. [PubMed: 17321816]

64. Wu C, Lin K, Chen H, Chen I, Hong W. Effects of modified constraint-induced movement therapy on movement kinematics and daily function in patients with stroke: a kinematic study of motor control mechanisms. Neurorehabil Neural Repair. 2007; 21(5):460-6. [PubMed: 17601803]

65. Kim D, Cho Y, Hong J, Song J, Chung H, Bai D, et al. Effect of constraint-induced movement therapy with modified opposition restriction orthosis in chronic hemiparetic patients with stroke. NeuroRehabilitation. 2008; 23(3):239-44. [PubMed: 18560140]

66. Azab M, Al-Jarrah M, Nazzal M, Maayah M, Sammour M, Jamous M. Effectiveness of constraintinduced movement therapy (CIMT) as home-based therapy on Barthel Index in patients with chronic stroke. Top Stroke Rehabil. 2009; 16(3):207-11. [PubMed: 19632965]

67. Brogärdh C, Vestling M, Sjolund B. Shortened constraint-induced movement therapy in subacute stroke - no effect of using a restraint: a randomized controlled study with independent observers. J Rehabil Med. 2009; 41(4):231-6. [PubMed: 19247541]

68. Brogärdh C, Lexell J. A 1-year follow-up after shortened constraint-induced movement therapy with and without mitt poststroke. Arch Phys Med Rehabil. 2010; 91(3):460-4. [PubMed: 20298840]

69. Hammer A, Lindmark B. Is forced use of the paretic upper limb beneficial? A randomized pilot study during subacute post-stroke recovery. Clin Rehabil. 2009; 23(5):424-33. [PubMed: 19321522]

70. Lin K, Chang Y, Wu C, Chen Y. Effects of constraint-induced therapy versus bilateral arm training on motor performance, daily functions, and quality of life in stroke survivors. Neurorehabil Neural Repair. 2009; 23(5):441-8. [PubMed: 19118130]

71. Lin K, Wu C, Liu J, Chen Y, Hsu C. Constraint-induced therapy versus dose-matched control intervention to improve motor ability, basic/extended daily functions, and quality of life in stroke. Neurorehabil Neural Repair. 2009; 23(2):160-5. [PubMed: 18981188]

72. Lin K, Chung H, Wu C, Liu H, Hsieh Y, Chen I, et al. Constraint-induced therapy versus control intervention in patients with stroke: a functional magnetic resonance imaging study. Am J Phys Med Rehabil. 2010; 89(3):177-85. [PubMed: 20173425]

73. Sun S, Hsu C, Sun H, Hwang C, Yang C, Wang J. Combined botulinum toxin type A with modified constraint-induced movement therapy for chronic stroke patients with upper extremity spasticity: a randomized controlled study. Neurorehabil Neural Repair. 2010; 24(1):34-41. [PubMed: 19729582]

74. Wu C, Hsieh Y, Lin K, Chuang L, Chang Y, Liu H, et al. Brain reorganization after bilateral arm training and distributed constraint-induced therapy in stroke patients: a preliminary functional magnetic resonance imaging study. Chang Gung Med J. 2010; 33(6):628-38. [PubMed: 21199608]

75. Meier Khan C, Oesch P, Gamper U, Kool J, Beer S. Potential effectiveness of three different treatment approaches to improve minimal to moderate arm and hand function after stroke--a pilot randomized clinical trial. Clin Rehabil. 2011; 25(11):1032-41. [PubMed: 21788267]

76. Wang Q, Zhao J, Zhu Q, Li J, Meng P. Comparison of conventional therapy, intensive therapy and modified constraint-induced movement therapy to improve upper extremity function after stroke. J Rehabil Med. 2011; 43(7):619-25. [PubMed: 21603848]

77. Wu C, Chuang L, Lin K, Chen H, Tsay P. Randomized trial of distributed constraint-induced therapy versus bilateral arm training for the rehabilitation of upper-limb motor control and function after stroke. Neurorehabil Neural Repair. 2011; 25(2):130-9. [PubMed: 20947493]

78. Brunner I, Skouen J, Strand L. Is modified constraint-induced movement therapy more effective than bimanual training in improving arm motor function in the subacute phase post stroke? A randomized controlled trial. Clin Rehabil. 2012; 26(12):1078-86. [PubMed: 22561098] 
79. Krawczyk M, Sidaway M, Radwanska A, Zaborska J, Ujma R, Czlonkowska A. Effects of sling and voluntary constraint during constraint-induced movement therapy for the arm after stroke: a randomized, prospective, single-centre, blinded observer rated study. Clin Rehabil. 2012; 26(11): 990-8. [PubMed: 22584730]

80. Wu C, Chen Y, Chen H, Lin K, Yeh I. Pilot trial of distributed constraint-induced therapy with trunk restraint to improve poststroke reach to grasp and trunk kinematics. Neurorehabil Neural Repair. 2012; 26(3):247-55. [PubMed: 21903975]

81. Wu C, Chen Y, Lin K, Chao C, Chen Y. Constraint-induced therapy with trunk restraint for improving functional outcomes and trunk-arm control after stroke: a randomized controlled trial. Phys Ther. 2012; 92(4):483-92. [PubMed: 22228607]

82. van Delden A, Peper C, Nienhuys K, Zijp N, Beek P, Kwakkel G. Unilateral versus bilateral upper limb training after stroke: the Upper Limb Training After Stroke Clinical trial. Stroke. 2013; 44(9): 2613-6. [PubMed: 23868279]

83. Wolf S, Maddy D, Newton H, Blanton S, Winstein C, Zhang Q. The EXCITE Trial: relationship of intensity of Constraint Induced Movement Therapy to improvement in the Wolf Motor Function Test. Restorative neurology and neuroscience. 2007; 25(5-6):549. [PubMed: 18334772]

84. van Delden A, Peper C, Beek P, Kwakkel G. Unilateral versus bilateral upper limb exercise therapy after stroke: a systematic review. J Rehabil Med. 2012; 44(2):106-17. [PubMed: 22266762]

85. Park S, Butler A, Cavalheiro V, Alberts J, Wolf S. Changes in serial optical topography and TMS during task performance after constraint-induced movement therapy in stroke: a case study. Neurorehabil Neural Repair. 2004; 18(2):95-105. [PubMed: 15228805]

86. Butler A, Wolf S. Putting the brain on the map: use of transcranial magnetic stimulation to assess and induce cortical plasticity of upper-extremity movement. Phys Ther. 2007; 87(6):719-36. [PubMed: 17429003]

87. Liepert J, Miltner W, Bauder H, Sommer M, Dettmers C, Taub E, et al. Motor cortex plasticity during constraint-induced movement therapy in stroke patients. Neuroscience letters. 1998; 250(1):5-8. [PubMed: 9696052]

88. Shmuelof L, Krakauer J. Are we ready for a natural history of motor learning? Neuron. 2011; 72(3):469-76. [PubMed: 22078506]

89. Rijntjes M, Hamzei F, Glauche V, Saur D, Weiller C. Activation changes in sensorimotor cortex during improvement due to CIMT in chronic stroke. Restorative neurology and neuroscience. 2011; 29(5):299-310. [PubMed: 21697588]

90. Laible M, Grieshammer S, Seidel G, M R, Weiller C, Hamzei F. Association of activity changes in the primary sensory cortex with successful motor rehabilitation of the hand following stroke. Neurorehabil Neural Repair. 2012; 26(7):881-8. [PubMed: 22396499]

91. Buma F, Lindeman E, Ramsey N, Kwakkel G. Functional neuroimaging studies of early upper limb recovery after stroke: a systematic review of the literature. Neurorehabil Neural Repair. 2010; 24(7):589-608. [PubMed: 20439501]

92. Buma F, Kwakkel G, Ramsey N. Understanding upper limb recovery after stroke. Restorative neurology and neuroscience. 2013

93. Kitago T, Liang J, Huang V, Hayes S, Simon P, Tenteromano L, et al. Improvement after constraint-induced movement therapy: recovery of normal motor control or task-specific compensation? Neurorehabil Neural Repair. 2013; 27(2):99-109. [PubMed: 22798152]

94. van Kordelaar J, van Wegen E, Nijland R, Daffertshofer A, Kwakkel G. Understanding adaptive motor control of the paretic upper limb early poststroke: The EXPLICIT-stroke program. Neurorehabil Neural Repair. 2013; 27(9):854-63. [PubMed: 23884015]

95. van Kordelaar J, van Wegen E, Kwakkel G. The impact of time on quality of motor control of the paretic upper limb after stroke. Arch Phys Med Rehabil. 2013

96. van Dokkum L, Hauret I, Mottet D, Froger J, Metrot J, Laffont I. The contribution of kinematics in the assessment of upper limb motor recovery early after stroke. Neurorehabil Neural Repair. 2013

97. Metrot J, Froger J, Hauret I, Mottet D, Van Dokkum L, Laffont I. Motor recovery of the ipsilesional upper limb in subacute stroke. Arch Phys Med Rehabil. 2013; 94(11):2283-90. [PubMed: 23796686] 
98. Kwakkel G, Kollen B, Twsk J. Impact of time on improvement of outcome after stroke. Stroke. 2006; 37(9):2348-53. [PubMed: 16931787]

99. Duncan P, Goldstein L, Matchar D, Divine G, Feussner J. Measurement of motor recovery after stroke. Outcome assessment and sample size requirements. Stroke. 1992; 23(8):1084-9. [PubMed: 1636182]

100. Murphy T, Corbett D. Plasticity during stroke recovery: from synapse to behaviour. Nature reviews Neuroscience. 2009; 10(12):861-72.

101. Taub E, Crago J, Uswatte G. Constraint induced movement therapy: a new approach to teatment in physical medicine. Rehabil Psychol. 1998; 43(2):152-70.

102. Stinear C, Barber P, Smale P, Coxon J, Fleming M, Byblow W. Functional potential in chronic stroke patients depends on corticospinal tract integrity. Brain: a journal of neurology. 2007; 130(Pt 1):170-80. [PubMed: 17148468]

103. Stinear C. Prediction of recovery of motor function after stroke. Lancet Neurol. 2010; 9(12): 1228-32. [PubMed: 21035399]

104. Smania N, Paolucci S, Tinazzi M, Borghero A, Manganotti P, Fiaschi A, et al. Active finger extension: a simple movement predicting recovery of arm function in patients with acute stroke. Stroke. 2007; 38(3):1088-90. [PubMed: 17255546]

105. Nijland R, van Wegen E, Harmeling-van der Wel B, Kwakkel G. Presence of finger extension and shoulder abduction within 72 hours after stroke predicts functional recovery: early prediction of functional outcome after stroke: the EPOS cohort study. Stroke. 2010; 41(4):745-50. [PubMed: 20167916]

106. Stinear C, Barber P, Petoe M, Anwar S, Byblow W. The PREP algorithm predicts potential for upper limb recovery after stroke. Brain: a journal of neurology. 2012; 135(Pt 8):2527-35. [PubMed: 22689909]

107. Fritz S, Light K, Patterson T, Behrman A, Davis S. Active finger extension predicts outcomes after constraint-induced movement therapy for individuals with hemiparesis after stroke. Stroke. 2005; 36(6):1172-7. [PubMed: 15890987]

108. Mark V, Taub E. Constraint-induced movement therapy for chronic stroke hemiparesis and other disabilities. Restorative neurology and neuroscience. 2004; 22(3-5):317-36. [PubMed: 15502259]

109. Lang C, Edwards D, Birkenmeier R, Dromerick A. Estimating minimal clinically important differences of upper-extremity measures early after stroke. Arch Phys Med Rehabil. 2008; 89(9): 1693-700. [PubMed: 18760153]

110. Van der Lee J, Beckerman H, Lankhorst G, Bouter L. The responsiveness of the Action Research Arm test and the Fugl-Meyer Assessment scale in chronic stroke patients. J Rehabil Med. 33(3): 110-13. [PubMed: 11482350]

111. Peurala S, Kantanen M, Sjögren T, Paltamaa J, Karhula M, Heinonen A. Effectiveness of constraint-induced movement therapy on activity and participation after stroke: a systematic review and meta-analysis of randomized controlled trials. Clin Rehabil. 2012; 26(3):209-23. [PubMed: 22070990]

112. Dwan K, Gamble C, Williamson PR, Kirkham J. Systematic review of the empirical evidence of study publication bias and outcome reporting bias - an updated review. PloS one. 2013; 8(7):e66844. [PubMed: 23861749]

113. Riley R, Lambert P, Abo-Zaid G. Meta-analysis of individual participant data: rationale, conduct, and reporting. BMJ (Clinical research ed). 2010; 340:c221.

114. Higgins, J.; Green, S. Chapter 9: Analysing data and undertaking meta-analyses. In: Higgins, J.; Green, S., editors. Cochrane Handbook for Systematic Reviews of Interventions Version 510. The Cochrane Collaboration; 2009. updated March 2011

115. Biernaskie J, Chernenko G, Corbett D. Efficacy of rehabilitative experience declines with time after focal ischemic brain injury. The Journal of neuroscience: the official journal of the Society for Neuroscience. 2004; 24(5):1245-54. [PubMed: 14762143]

116. Ploughman M, Windle V, MacLellan C, White N, Dore J, Corbett D. Brain-derived neurotrophic factor contributes to recovery of skilled reaching after focal ischemia in rats. Stroke. 2009; 40(4): 1490-5. [PubMed: 19164786] 
117. Zhao S, Zhao M, Xiao T, Jolkkonen J, Zhao C. Constraint-induced movement therapy overcomes the intrinsic axonal growth-inhibitory signals in stroke rats. Stroke. 2013; 44(6):1698-705. [PubMed: 23632976]

118. Joo H, Hyun J, Kim T, Chae S, Lee Y, Lee S. Influence of constraint-induced movement therapy upon evoked potentials in rats with cerebral infarction. The European journal of neuroscience. 2012; 36(12):3691-7. [PubMed: 23043504]

119. Robertson I, Murre J. Rehabilitation of brain damage: brain plasticity and principles of guided recovery. Psychological bulletin. 1999; 125(5):544-75. [PubMed: 10489541]

120. Whishaw I, Alaverdashvili M, Kolb B. The problem of relating plasticity and skilled reaching after motor cortex stroke in the rat. Behavioural brain research. 2008; 192(1):124-36. [PubMed: 18282620]

121. Janssen H, Speare S, Spratt N, Sena E, L A, Hannan A, et al. Exploring the efficacy of constraint in animal models of stroke: meta-analysis and systematic review of the current evidence. Neurorehabil Neural Repair. 2013; 27(1):3-12. [PubMed: 22714123]

122. Bland S, Schallert T, Strong R, Aronowski J, Grotta J, Feeney D. Early exclusive use of the affected forelimb after moderate transient focal ischemia in rats: functional and anatomic outcome. Stroke. 2000; 31(5):1144-52. [PubMed: 10797179]

123. Bland S, Pillai R, Aronowski J, Grotta J, Schallert T. Early overuse and disuse of the affected forelimb after moderately severe intraluminal suture occlusion of the middle cerebral artery in rats. Behavioural brain research. 2001; 126(1-2):33-41. [PubMed: 11704249]

124. Kozlowski D, James D, Schallert T. Use-dependent exaggeration of neuronal injury after unilateral sensorimotor cortex lesions. The Journal of neuroscience: the official journal of the Society for Neuroscience. 1996; 16(15):4776-86. [PubMed: 8764664]

125. Medical Advisory Secretariat HQO. Constraint-induced movement therapy for rehabilitation of arm dysfunction after stroke in adults: an evidence-based analysis. Ont Health Technol Assess Ser. 2011; 11(6):1-58.

126. Hosomi M, Koyama T, Takebayashi T, Terayama S, Kodama N, Matsumoto K, et al. A modified method for constraint-induced movement therapy: a supervised self-training protocol. Journal of stroke and cerebrovascular diseases: the official journal of National Stroke Association. 2012; 21(8):767-75. [PubMed: 21596584] 
Panel 1

\section{Search strategy and selection criteria}

We identified relevant publications by searching PubMed, EBSCO host/Excerpta Medica Databank (EMBASE), EBSCOhost/Cumulative Index of Nursing and Allied Health Literature (CINAHL), Wiley/Cochrane Library (Cochrane Database of Systematic Reviews [CDSR], Cochrane Central Register of Controlled Trials [CENTRAL], Cochrane Methodology Register [CMR], Database of Abstracts of Reviews of Effects [DARE], Health Technology Assessment Database [HTA], NHS Economic Evaluation Database [EED]) and Physiotherapy Evidence Database (PEDro) (all databases last searched 24-09-2013 by JMV). The indexing terms and free-text terms with synonyms and related terms in the title or abstract used were: "stroke" and "physical restraint" or "constraint-induced movement therapy" or "forced use" or "immobilization" or "learned nonuse" and "randomized controlled trial" or "reviews" (supplementary web appendix 2). One reviewer (JMV) screened the titles, abstracts, if relevant full-text publications, and references of included RCTs. The inclusion criteria were: (1) adult stroke patients; (2) RCT design, including those with a two-group parallel, multi-arm parallel, crossover, cluster or factorial design; (3) the experimental intervention conformed to the definitions of original CIMT, mCIMT, or FU; (4) the comparator was usual care, another intervention, the same intervention with a different dose, or no intervention; (5) outcomes were measured post intervention and/or at follow-up; and (6) publication in the English, French, German, or Dutch. 


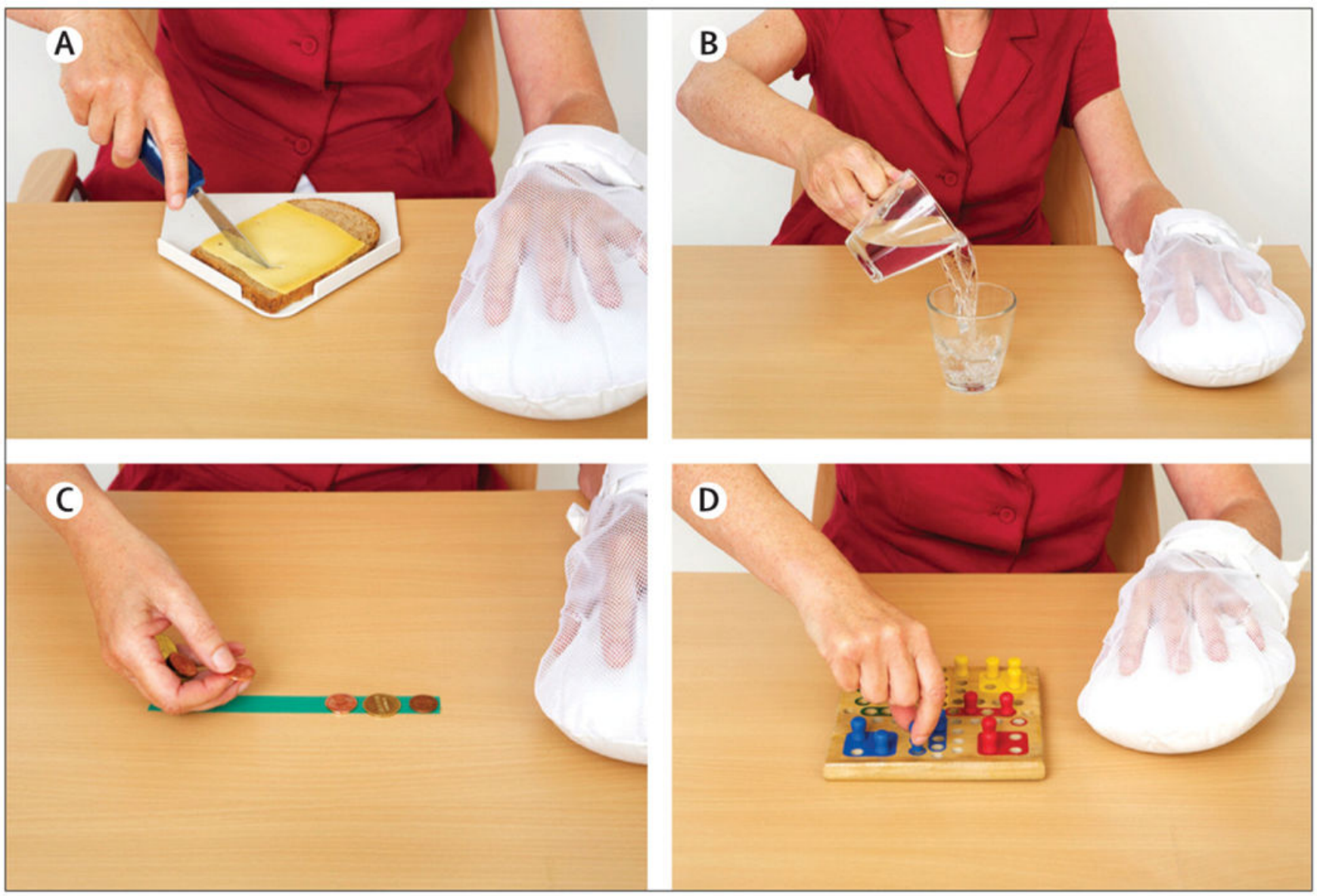

Figure 1. Constraint-induced movement therapy

Illustration of task-oriented practice with the paretic limb in a Constrained Induced

Movement Therapy session. Use of the non-affected limb is restricted by a padded mitt. 


\begin{tabular}{|c|c|c|c|c|c|}
\hline Intervention & $\begin{array}{l}\text { Number of } \\
\text { comparisons }\end{array}$ & Number of participants $(E / C)$ & $P^{2}$ & $\begin{array}{l}\text { Overall effect sizes } 95 \% \mathrm{Cl} \\
\text { (based on adjusted Hedges' g) }\end{array}$ & Statistical power \\
\hline \multicolumn{6}{|l|}{ Arm motor function } \\
\hline Original CIMT & .. & .. & .. & & .. \\
\hline 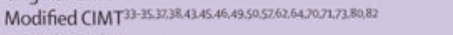 & 24 & $228 / 295$ & $72 \%$ & & $0.98^{*}$ \\
\hline Forced use therapy & $\cdots$ & . &. & & 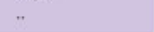 \\
\hline \multicolumn{6}{|l|}{ Grip strength } \\
\hline Original CIMT & 1 & $106 / 116$ & 0 & & 0.28 \\
\hline Modified CIMT ${ }^{30.42}$ & 2 & $50 / 49$ & 0 & & 0.09 \\
\hline Forced use therapy &. & - &. & &. \\
\hline \multicolumn{6}{|l|}{ Muscle tone } \\
\hline Original CIMT & .. & .. & .. & & .. \\
\hline Modified CIMTS5/3 & 2 & $46 / 45$ & 0 & - & 0.37 \\
\hline Forced use therapy & $\therefore$ & . & $\because$ & & . \\
\hline \multicolumn{6}{|l|}{ Sensibility } \\
\hline Original CIMT & .. &. & .. & & . \\
\hline Modified CIMT ${ }^{4682}$ & 3 & $31 / 42$ & $30 \%$ & & 0.08 \\
\hline Forced use therapy &. & - & 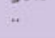 & &. \\
\hline \multicolumn{6}{|l|}{ Pain } \\
\hline Original CIMT &. & .. & .. & & .. \\
\hline Modified CIMT ${ }^{4675}$ & 3 & $23 / 37$ & 0 & & 0.05 \\
\hline Forced use therapy & * & . &. & &. \\
\hline \multicolumn{6}{|l|}{ Arm-hand activities } \\
\hline Original $\mathrm{CIMT}^{28}$ & 1 & $106 / 116$ & 0 & $\Rightarrow$ & $0.93^{*}$ \\
\hline Modified CIMT ${ }^{3233}-43.65-4751-567375,78.80-82$ & 40 & $429 / 545$ & $49 \%$ & $\bullet$ & $1.00^{*}$ \\
\hline Forced use therapy &. & . & 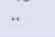 & & $*$ \\
\hline \multicolumn{6}{|l|}{ Self-reported amount of arm-hand use } \\
\hline Original $\mathrm{CIMT}^{28}$ & 1 & $106 / 116$ & 0 & $\Rightarrow$ & $0.93^{*}$ \\
\hline 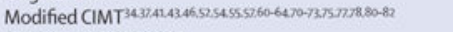 & 30 & $364 / 475$ & $75 \%$ & $<$ & $1.00^{*}$ \\
\hline Forced use therapy $y^{6769}$ & 2 & $25 / 27$ & 0 & & 0.08 \\
\hline \multicolumn{6}{|l|}{ Self-reported quality of arm-hand use } \\
\hline Original CIMT ${ }^{28}$ & 1 & $106 / 116$ & 0 & - & $0.98^{*}$ \\
\hline 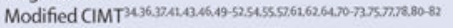 & 34 & $397 / 516$ & $51 \%$ & & 1.00 \\
\hline Forced use therapy $y^{67.6279}$ & 3 & $49 / 50$ & 0 & & 0.07 \\
\hline \multicolumn{6}{|l|}{ Basic ADL } \\
\hline Original CIMT &. &. & .. & & .. \\
\hline Modified CIMT ${ }^{41,452525462-646671}$ & 11 & $157 / 176$ & 0 & $<$ & 0.63 \\
\hline Forced use therapy & .* & - & .. & &. \\
\hline \multicolumn{6}{|l|}{ Extended ADL } \\
\hline Original CIMT & .. & .. & .. & & ." \\
\hline Modified CIMT & .. & .. & .. & & " \\
\hline Forced use therapy & .. & -. & .. & & " \\
\hline \multicolumn{6}{|l|}{ Quality of life related to hand function } \\
\hline Original CIMT & . & .. & .. & & . \\
\hline Modified CIMT $53.81,82$ & 8 & $64 / 100$ & 0 & & 0.06 \\
\hline Forced use therapy & . & 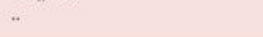 &. & &. \\
\hline \multicolumn{6}{|l|}{ Quality of life related to $A D L$} \\
\hline Original CIMT & " & -. & . & & 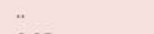 \\
\hline Modified CIMT ${ }^{81,82}$ & 4 & $40 / 72$ & 0 & 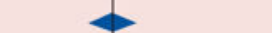 & 0.05 \\
\hline \multirow[t]{3}{*}{ Forced use therapy } & .. & - & 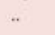 & & $\cdot$ \\
\hline & & & & $\underset{-1}{\Gamma}$ & \\
\hline & & & ors con & $\overrightarrow{\text { Favours experi }}$ & nental group \\
\hline
\end{tabular}

Figure 2. Forest plot of constraint-induced movement therapy post intervention

This figure displays the summarized effects of original CIMT, modified CIMT and forced use post intervention, classified according to the International Classification of Functioning, disability and health (ICF; World Health Organization). Please consult supplementary web appendix 5 for Hedges' g (95\% CI) in numbers. Background colors represent the different ICF-categories, body functions (pink), activities (blue), and participation (orange). The italic numbers in the statistical power column indicate sufficient power $(1-\beta \searrow 0.80)$. 95\% CI, 95\% 
Confidence interval; ADL, Activities of daily living; C, Control group; CIMT, Constraintinduced movement therapy; E, Experimental group; N/A, Not applicable.

References: (a) 33-35, 37, 38, 43, 45, 46, 49, 50, 57, 62, 64, 70, 71, 73, 80, 82; (b) 39, 42; (c) 55,73 ; (d) 46,82 ; (e) 46,75 ; (f) 28 ; (g) $31,33-43,45-47,51-56,73,75-78,80-82$; (h) 28; (i) $34,37,41,43,46,52,54,55,57,61-64,70-73,75,77,78,80-82$; (j) 67, 69; (k) 28 ; (l) $34,36,37,41,43,46,49-52,54,55,57,61,62,64,70-73,75,77,78,80-82$; (m) 67, 69, 79 ; (n) $41,47,52,54,62-64,66,71$; (o) $53,81,82$; (p) $81,82$. 


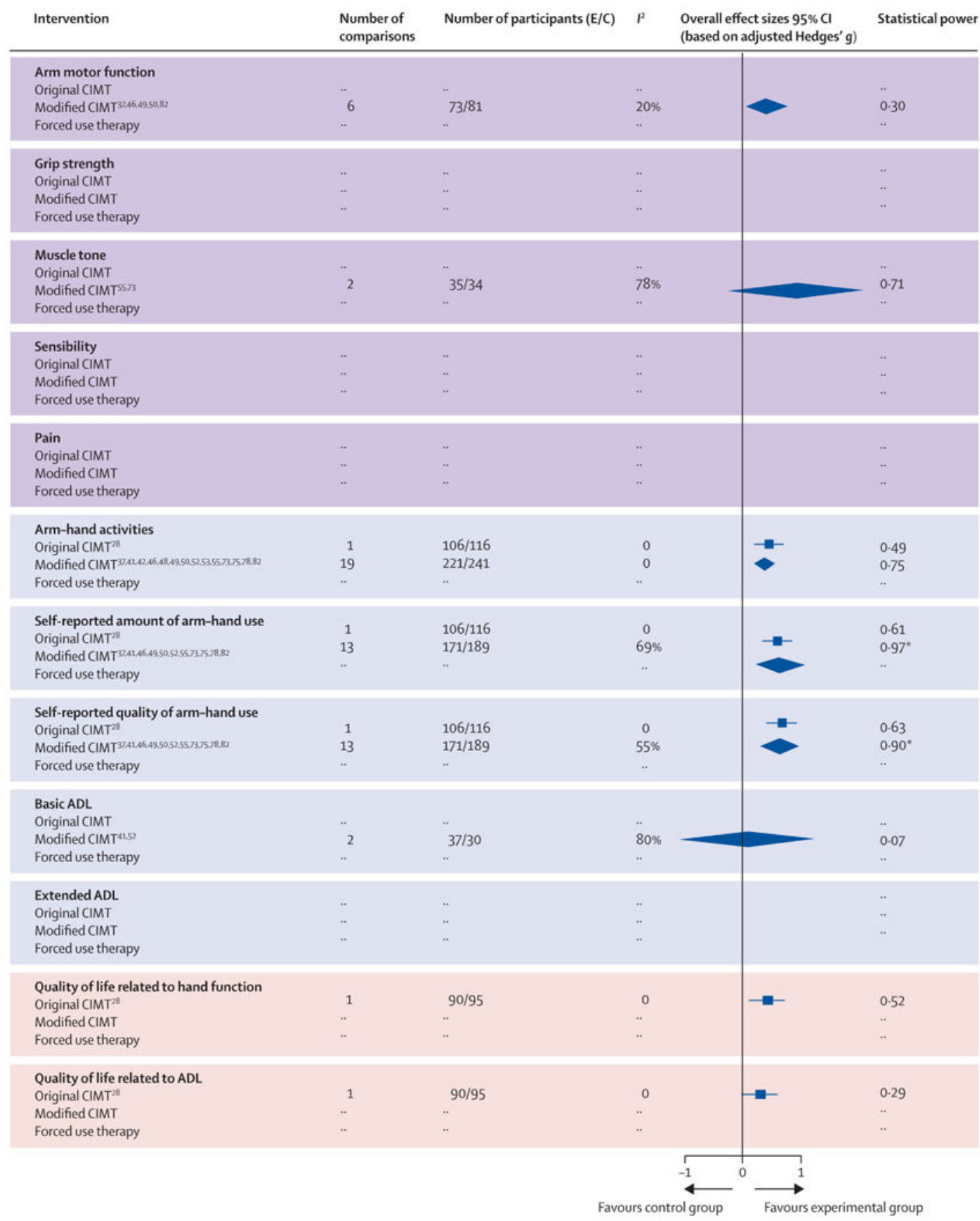

Figure 3. Forest plot of constraint-induced movement therapy in the long term

This figure displays the summarized effects of original CIMT, modified CIMT and forced use in the long term (4-5 months), classified according to the International Classification of Functioning, disability and health (ICF; World Health Organization). Please consult supplementary web appendix 6 for Hedges' g $(95 \% \mathrm{CI})$ in numbers. Background colors represent the different ICF-categories, body functions (pink), activities (blue), and participation (orange). The italic numbers in the statistical power column indicate sufficient power $(1-\beta \searrow 0.80)$. 95\% CI, 95\% Confidence interval; ADL, Activities of daily living; C, 
Control group; CIMT, Constraint-induced movement therapy; E, Experimental group; N/A, Not applicable.

References: (a) 37, 46, 49, 50, 82; (b) 55, 73; (c) 28; (d) 37, 41, 42, 46, 48, 49, 50, 52, 53, $55,73,75,78,82$; (e) 28 ; (f) $37,41,46,49,50,52,55,73,75,78,82$; (g) 28; (h) 37, 41, 46, 49, 50, 52, 55, 73, 75, 78, 82; (i) 41, 52; (j) 28; (k) 28 . 
Table 1

Summary of evidence for constraint-induced movement therapy according to the ICF

In this table, the evidence for original CIMT, modified CIMT, and forced use post intervention and in the long term (4-5 months) are summarized according to the International classification of function, disability, and health model (ICF). $\checkmark$, beneficial or likely to be beneficial based on significant positive summary effect sizes; $\times$, uncertain benefit based on non-significant summary effect sizes; ?, unknown effect based on the inability to statistically pool data of RCTs; *, only beneficial or likely to be beneficial within the first 3 months post stroke. ADL, Activities of daily living; AOU, Self-reported amount of arm-hand use in daily life; CIMT, Constraint-induced movement therapy; FU, Forced use; QoL, Quality of life; QOM, Self-reported quality of arm-hand movement in daily life.

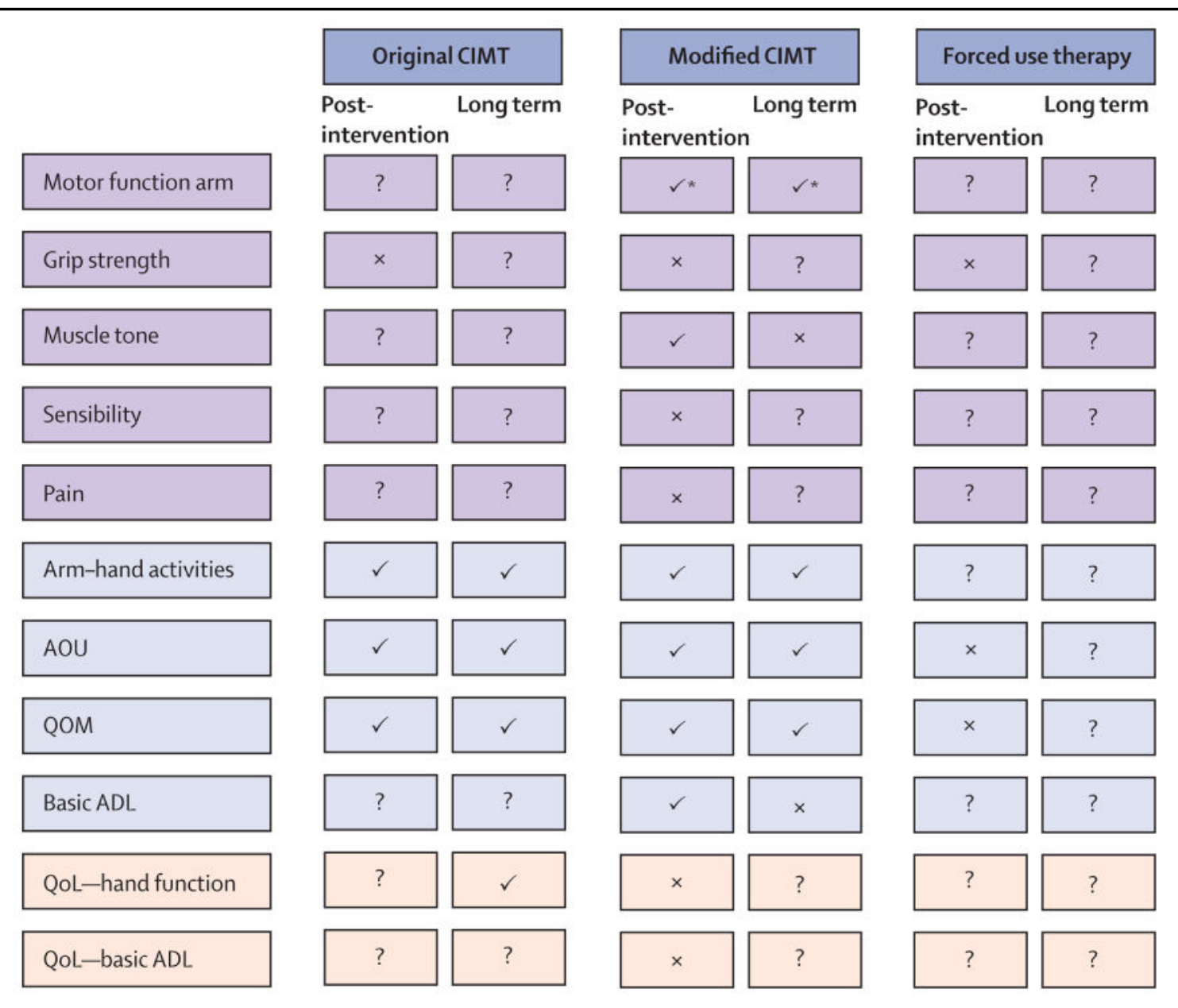

